\title{
Emergence and spread of the potential variant of interest (VOI) B.1.1.519 of SARS-CoV-2 predominantly present in Mexico
}

\author{
Abril Paulina Rodríguez-Maldonado ${ }^{1}$. Joel Armando Vázquez-Pérez ${ }^{3}$ (1) - Alberto Cedro-Tanda ${ }^{2}$ (D) \\ Blanca Taboada ${ }^{4}$. Celia Boukadida ${ }^{5}$. Claudia Wong-Arámbula ${ }^{1}$ Tatiana Ernestina Nuñez-García ${ }^{1}$. \\ Natividad Cruz-Ortiz ${ }^{1}$. Gisela Barrera-Badillo ${ }^{1}$ (1) - Lucía Hernández-Rivas ${ }^{1}$ - Irma López-Martínez ${ }^{1}$.

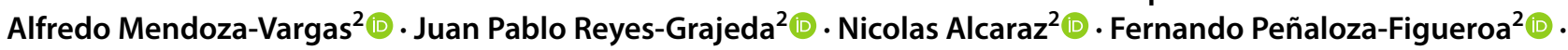 \\ Dulibeth Gonzalez-Barrera ${ }^{2}$ - Daniel Rangel-DeLeon ${ }^{2} \cdot$ Luis Alonso Herrera-Montalvo $^{2} \mathbb{D}$. \\ Fidencio Mejía-Nepomuceno ${ }^{3}$ - Alejandra Hernández-Terán ${ }^{3}$ - Mario Mújica-Sánchez ${ }^{3}$ - Eduardo Becerril-Vargas ${ }^{3}$. \\ José Arturo Martínez-Orozco ${ }^{3} \cdot$ Rogelio Pérez-Padilla $^{3}$. Jorge Salas-Hernández ${ }^{3}$. Alejandro Sanchez-Flores ${ }^{4}$ (D) \\ Pavel Isa ${ }^{4}$ - Margarita Matías-Florentino ${ }^{5}$ - Santiago Ávila-Ríos ${ }^{5}$. José Esteban Muñoz-Medina ${ }^{6}$ (D) \\ Concepción Grajales-Muñiz $z^{6}$. Angel Gustavo Salas-Lais ${ }^{6}$ (D) Andrea Santos Coy-Arechavaleta ${ }^{6}$. \\ Alfredo Hidalgo-Miranda ${ }^{2}$. Carlos F. Arias ${ }^{5}$ (D) José Ernesto Ramírez-González ${ }^{1}$ (I)
}

Received: 13 April 2021 / Accepted: 27 June 2021 / Published online: 27 August 2021

(c) The Author(s), under exclusive licence to Springer-Verlag GmbH Austria, part of Springer Nature 2021

\begin{abstract}
SARS-CoV-2 variants emerged in late 2020, and at least three variants of concern (B.1.1.7, B.1.351, and P1) have been reported by WHO. These variants have several substitutions in the spike protein that affect receptor binding; they exhibit increased transmissibility and may be associated with reduced vaccine effectiveness. In the present work, we report the identification of a potential variant of interest, harboring the mutations T478K, $\mathrm{P} 681 \mathrm{H}$, and T732A in the spike protein, within the newly named lineage B.1.1.519, that rapidly outcompeted the preexisting variants in Mexico and has been the dominant virus in the country during the first trimester of 2021.
\end{abstract}

Viral mutation is a natural and expected event generated during genomic replication and interaction with the host, resulting in the establishment of genetic groups, also called

Handling Editor: Sheela Ramamoorthy.

Abril Paulina Rodríguez-Maldonado, Alberto Cedro-Tanda, Joel Armando Vázquez-Pérez, Blanca Taboada and Celia Boukadida contributed equally to this work. The order of the other authors was randomly assigned.

Alfredo Hidalgo-Miranda

ahidalgo@inmegen.gob.mx

Carlos F. Arias

arias@ibt.unam.mx

José Ernesto Ramírez-González

ernesto.ramirez@salud.gob.mx

1 Dirección General de Epidemiología, Instituto de Diagnóstico y Referencia Epidemiológicos “Dr. Manuel Martínez Báez, Mexico City, Mexico

2 Instituto Nacional de Medicina Genómica, Mexico City, Mexico lineages. The latter differ from each other by specific mutations that accumulate over time, causing the appearance of variants. A variant can be defined as a virus with specific genetic mutations that differ from the original virus, in some cases reflecting the adaptation of SARS-CoV-2 to its new human host. Although the majority of mutations in the SARS-CoV-2 genome are expected to be neutral or deleterious, some mutations can confer a selective advantage and may be associated with enhanced fitness, increased

3 Instituto Nacional de Enfermedades Respiratorias, Mexico City, Mexico

4 Instituto de Biotecnología, Universidad Nacional Autónoma de México, Cuernavaca, Morelos, Mexico

5 Centro de Investigación en Enfermedades Infecciosas, Instituto Nacional de Enfermedades Respiratorias "Ismael Cosío Villegas", Mexico City, Mexico

6 Instituto Mexicano del Seguro Social, Mexico City, Mexico 
infectivity, and/or immune evasion [1-3]. Importantly, the emergence and spread of variants associated with changes in transmission, virulence, and/or antigenicity can impact the evolution of the COVID-19 pandemic and might require appropriate public health actions and surveillance [4].

New SARS-CoV-2 variants are spreading rapidly around the world, becoming a public health concern. As of February 23, 2021, the Pan-American Health Organization (PAHO)/ World Health Organization (WHO) and Global Initiative on Sharing All Influenza Data (GISAID) reported the appearance of at least three variants of concern (VOCs) with characteristics that have implications for public health. Variant B.1.1.7 was identified for the first time in the United Kingdom in September 2020 [4, 5], and by December 2020 it represented $43 \%$ of the genomes sequenced, increasing to $82 \%$ in January 2021 and to $94 \%$ in February 2021 [6]. This variant is of growing concern, since it has been shown to be significantly more transmissible than other variants [7], and it is likely to have increased severity, based on hospitalization and fatality rates. Variant B.1.3 51 was detected for the first time in South Africa, in 64\% (261 of 411 genomes) of the sequences reported in December 2020, increasing to 75\% (99 of 132 genomes) in the next month [6]. Epidemiological data analysis estimated that this VOC is $50 \%$ more transmissible than the previous circulating variants. Finally, the P.1 variant was detected for the first time in Brazil in 47\% (61 of 130) of the viral genomes in December 2020, increasing to $74 \%$ (111 of 150 genomes) in the next month $[6,8]$. Of relevance, it has shown reduced neutralization by convalescent and post-vaccination sera [9]. These SARS-CoV-2 VOCs have acquired some of the same spike protein mutations independently, particularly E484K, N501Y, S477N, and K417T, which have been associated with increased viral transmission and/or decreased sensitivity to antibody neutralization [10].

In Latin America, with the exception of P.1 and P. 2 observed in Brazil, no other variants with the potential for rapid expansion have been reported so far [11]. Here, we report the identification of a potential VOI harboring the mutations $\mathrm{T} 478 \mathrm{~K}, \mathrm{P} 681 \mathrm{H}$, and $\mathrm{T} 732 \mathrm{~A}$ in the spike protein, within the newly named lineage B.1.1.519, derived from the B.1.1.222 lineage, that rapidly outcompeted the preexisting variants in Mexico and has been the dominant virus in the country during 2021.

Derived from genomic surveillance carried out in Mexico, 2,692 genomic sequences were obtained in this study and are part of the 3,156 sequences deposited in the GISAID from March 1, 2020 to March 21, 2021. As a result of the analysis of this set of sequences, we observed the presence of 91 Phylogenetic Assignment of Named Global Outbreak (PANGO) lineages, with B.1.1.519 (37.8\%), B.1 (13.9\%), B.1.1.222 (10.3\%), B.1.1 (5.7\%), B.1.609 (5.6\%), and B.1.243 (4.5\%) being the most prevalent. Libraries for whole genome sequencing of SARS-CoV-2 were generated using the protocol developed by the ARTIC Network (https://artic.network/2-protocols.html) or a long-ampliconbased method (https://pubmed.ncbi.nlm.nih.gov/32222995/).

A striking observation was the detection of the B.1.1.519 lineage in the USA, derived from B.1.1.222, which harbors the mutation T478K in the spike protein. This variant had not been detected in Mexico before October 2020, when it was found in Mexico City, and phylogeographic analysis suggested that the B.1.1.519 variant emerged around midSeptember 2020 [10]. In November 2020, 13\% (16/123) of the characterized cases of COVID-19 were caused by this variant, and in December, this proportion increased to $29.3 \%$ (97/331). In January 2021, the percentage of B.1.1.519 rose to $51.5 \%$ (229/445), increasing in incidence to $73.6 \%$ (808/1098) in February. On the other hand, a decreasing frequency of the B.1 lineage that had predominated in Mexico in 2020 was observed, going from $36.27 \%$ (284/783) between March and September to 2.37\% (26/1098) in February 2021.

In Mexico, since the identification of B.1.1.519 in November 2020, a total of 6419 genomic sequences have been reported with this lineage. The majority of them are from Mexico City and are spread throughout all country.

A detailed analysis of the samples from Mexico City indicated that, in November, this variant was present in $17.8 \%$ (13/73) of the cases, while in December 2020, this proportion increased to $47.5 \%$ (47/99). In January 2021, the variant was detected in $77.5 \%$ (138/178) of the cases and by February in $90.9 \%$ (349/384). This significant increase in the frequency of B.1.1.519 in Mexico City showed that it outcompeted preexisting variants between October 2020 and February 2021, and this increase was also observed in other regions of the country (Fig. 1), representing more than 50\% of the characterized viruses in some states during the first trimester of 2021. In particular, the variant was highly prevalent in Baja California Sur (51.3\%, 20/39), Guerrero (70\%, 21/30), Hidalgo (72.2\%, 13/18), Morelos (67.3\%, 33/49), State of Mexico (83.5\%, 76/91), Oaxaca $(51.3 \%, 19 / 37)$, Puebla $(78.5 \%, 77 / 98)$, Queretaro $(70.8 \%, 34 / 48)$, San Luis Potosi $(70 \%, 35 / 50)$, and Veracruz $(69.5 \%, 80 / 115)$.

This variant has also been detected in 17 countries on all five continents. In the Americas, it has been reported in Canada and the USA, and recently in Brazil, Chile, Aruba, Martinique, and Curazao [12]. However, this variant currently is not predominant in these countries.

The overall genome analysis of the viruses in the B.1.1.519 lineage showed the presence of 20 mutations in total, compared to the Wuhan-Hu-1 reference genome sequence (NCBI accession number MN908947). Eleven of these mutations are non-synonymous, and four of them are present in the spike protein. Notably, a T478K mutation is present in the receptor binding domain (RBD), where 


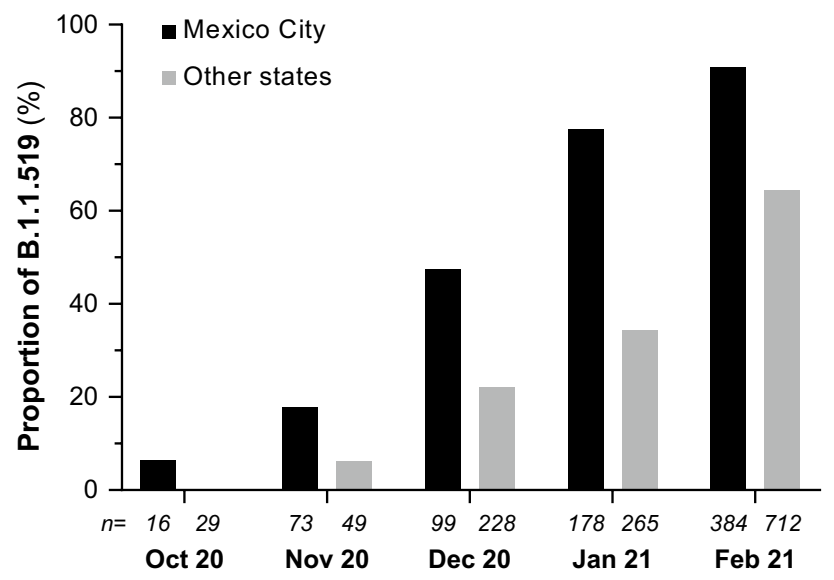

Fig. 1 Relative frequency of variant B.1.1.519 from October 2020 to February 2021. The monthly numbers of complete genome sequences (n) obtained from samples collected in Mexico City and other states of Mexico are indicated below each bar.

Table 1 Amino acid and nucleotide changes

\begin{tabular}{lll}
\hline Mutations & & Gene \\
\hline Nucleotide & Amino acid & \\
\hline C203T & - & \\
C222T & - & ORF1a \\
C241T & - & \\
C3037T & P141S & \\
C3140T & T492I & \\
C10029T & - & \\
C10954T & I49V & ORF1b \\
A11117G & & \\
C12789T & P323L & Spike \\
C14408T & - & \\
T19839C & - & \\
C21306T & T478K & \\
C22995A & D614G & \\
A23403G & P681H & \\
C23604A & T732A & \\
A23756G & - & \\
G28881A & R203K & \\
G28882A & G204R & \\
G28883C & - & \\
C29197T & & \\
\hline
\end{tabular}

The main amino acid substitutions are shown in bold

mutations have been shown to reduce the activity of some monoclonal antibodies [9]. All amino acid and nucleotide changes are listed in Table 1.

The current B.1.1.519 lineage, represented by the vast majority of the reported Mexican sequences, was first identified as a B.1.1.222 lineage. However, the presence of the mutations T478K, P681H, and T732A clearly differentiated it from this lineage, which does not contain these mutations, giving rise to the B.1.1.519 lineage. A phylogenomic analysis of genomic sequences using the Nextstrain tool showed that the viruses in the lineage B.1.1.519 (B.1.1.1.222+T478K+P681H+T732A) group independently of the lineage B.1.1.222 sequences, strongly suggesting that this variant should be classified as a variant of interest (VOI) (Fig. 2). On the other hand, viruses in the lineage B.1.1.519 are already grouped by the GISAID platform in an independent clade, invariably harboring the three mutations mentioned above.

An in silico analysis using different potent structures of related strains suggested that the position of the $\mathrm{T} 478 \mathrm{~K}$ mutation in the $\mathrm{S}$ protein is involved in antibody recognition and the receptor binding site [13]. In a deep mutational scanning of the SARS-CoV-2 receptor binding domain, the T478K mutation did not have a significant effect on folding or binding to human angiotensin-converting enzyme 2 (ACE2) [14]. However, this mutation may be involved in immune evasion, particularly escape from antibody neutralization [15]. The P681H mutation is one of the mutations found in the B.1.1.7 variant detected in the UK. According to the definitions described in the document issued by the WHO "Covid-19 Weekly Epidemiological Update" of February 25, 2021, with the special edition of "Proposed Working Definitions of SARS-CoV-2 Variants of Interest and Variants of Concern", we can consider the lineage B.1.1.519 a potential variant of interest [16].

Finally, two variants with interesting features were identified in this study: first, 13 sequences belonging to the B.1.1.222 lineage without the T478K mutation, but harboring the T732A mutation and the 69-70 deletion in the spike protein, the latter being a characteristic mutation of the B.1.1.7 VOC first detected in the UK; and second, 11 sequences corresponding to four lineages differing from B.1.1.519 (B.1, B.1.1.222, B.1.1.322, and B.1.323) but containing the same $\mathrm{T} 478 \mathrm{~K}, \mathrm{P} 681 \mathrm{H}$, and T732A mutations in the spike glycoprotein that are present in the variant B.1.1.519. Keeping track of the incidence of these two variants is recommended during genomic surveillance.

So far, we do not have experimental evidence to determine if the mutations described here could be associated with changes in transmission, virulence, and/or antigenicity or if they could have an impact on the severity of disease, reinfection rates, or vaccine effectiveness. For this reason, the importance of a genomic surveillance system, epidemiological studies, and experiments to assess the neutralization of viruses in lineage B.1.1.519 or any new variants are crucial for investigating the possible biological impact of the mutations in the context of public health. Fortunately, all COVID-19 virus variants that have emerged so far respond to the available, approved vaccines to some extent. 


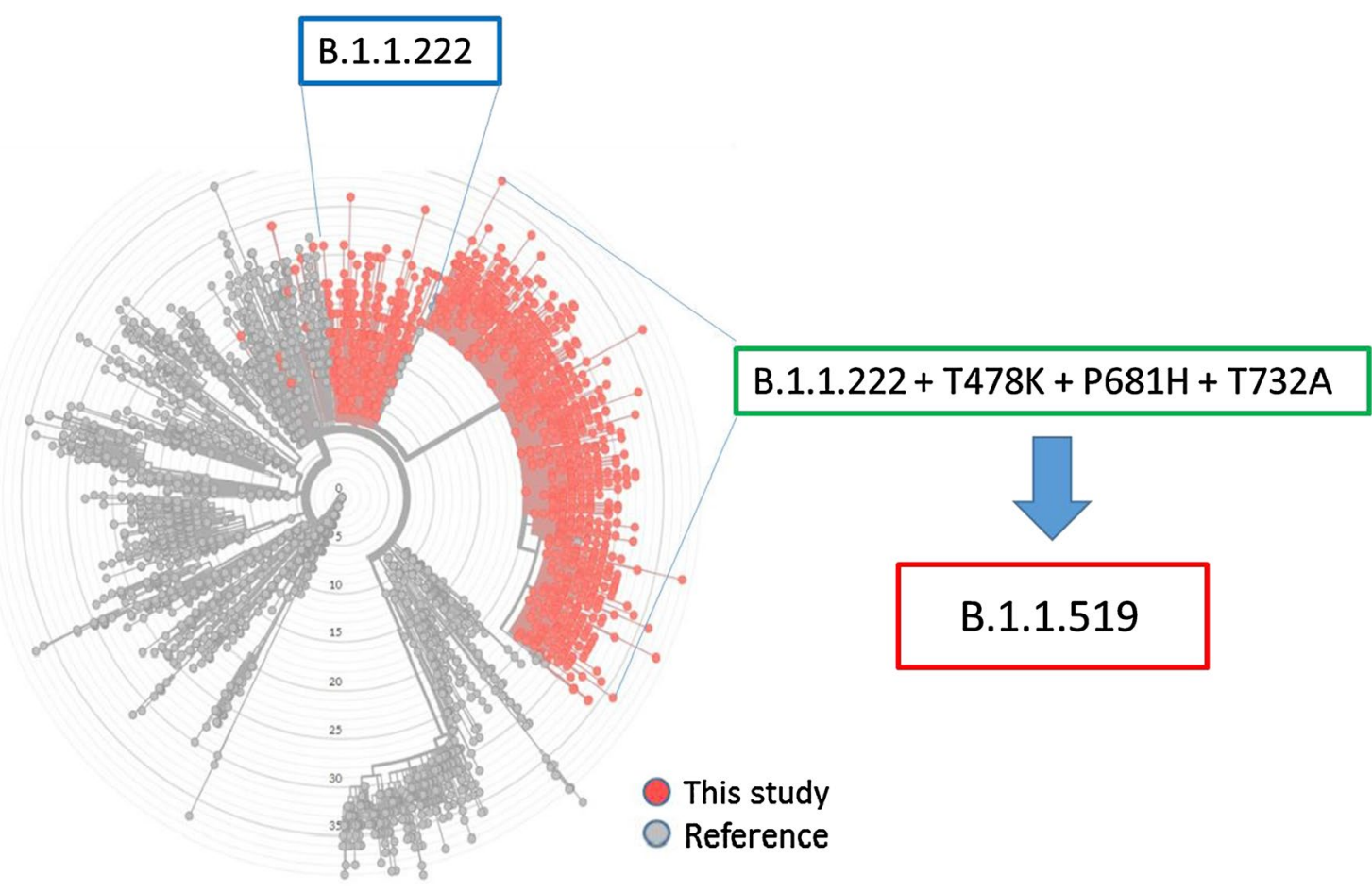

Fig. 2 Phylogenomic analysis of SARS CoV-2 sequences obtained in this study (red dots) and of reference sequences (gray dots), showing the clustering of variant B.1.1.519 (red box) independently of the lineage B.1.1.222 (blue box). Viruses in the B.1.1.519 lineage

Acknowledgements The authors express their gratitude to Vanessa Rivero, Ariadna Medina, Joaquin Quiroz, Sergio Rangel, Mayra Jiménez, David Fragoso, Nancy Miñoz, Edgar Mendieta, Fabiola Garcés and Adnan Araiza from InDRE for sequencing and bionformatical approach; to Ricardo Grande, Francisco Pulido and Gloria Vazquez from the "Unidad de Secuenciación Masiva y Bioinformática" of the "Laboratorio Nacional de Apoyo Tecnológico a las Ciencias Genómicas" (CONACyT no. 260481) for their support in sequencing services and also to the working team for molecular biology of the Central Laboratory of Epidemiology IMSS, for technical assistance. We thank all of the staff of the Technological Development and Molecular Research Unit of the Virology Department and of the Sample Control and Services Department at InDRE for technical assistance. The findings and conclusions in this report are those of the authors and do not necessarily represent the official opinion of the Ministry of Health of Mexico.

Funding This work was partially supported by the grants "Epidemiología Genómica de los Virus SARS-CoV-2 Circulantes en México" and "Caracterización de la diversidad viral y bacteriana" from the National Council for Science and Technology (CONACyT) of Mexico to C.F.A. and J.A.V.P., respectively, and also grants 057, 047, and 072 from the Ministry of Education, Science, Technology and Innovation (SECTEI) of Mexico City to C.F.A., L.H.M., and A.H.M., respectively.

\section{Declarations}

Conflict of interest The authors declare no conflict of interest. were initially classified within the B.1.1.222 lineage, and they contain the mutations T478K, P681H, and T732A (green box). The phylogenomic tree was powered a CC-BY-4.0 license and attribution of nextstrain.org.

Ethical approval This article does not contain any studies with human participants or animals performed by any of the authors.

\section{References}

1. Korber B, Fischer WM, Gnanakaran S et al (2020) Tracking changes in SARS-CoV-2 spike: evidence that D614G increases infectivity of the COVID-19 virus. Cell 182:812-827. https://doi. org/10.1016/j.cell.2020.06.043

2. Plante JA, Liu Y, Liu J et al (2020) Spike mutation D614G alters SARS-CoV-2 fitness. Nature 592:116-121. https://doi.org/10. 1038/s41586-020-2895-3

3. Li Q, Nie J, Wu J et al (2021) SARS-CoV-2 501Y.V2 variants lack higher infectivity but do have immune escape. Cell 184:23622371. https://doi.org/10.1016/j.cell.2021.02.042

4. Epidemiological update: occurrence of variants of SARS-CoV-2 in the Americas-20 January 2021. https://iris.paho.org/bitstream/ handle/10665.2/53219/EpiUpdate20January2021_eng.pdf?seque nce $=1 \&$ is Allowed $=y$. Accessed 29 Mar 2021

5. Weekly epidemiological update-23 February 2021. https://www. who.int/publications/m/item/weekly-epidemiological-update--23-february-2021. Accessed 29 Mar 2021

6. (2021) Tracking of variants. https://www.gisaid.org/hcov19-varia nts/. Accessed 29 Mar 2021

7. Davies NG, Abbott S, Barnard RC et al (2021) Estimated transmissibility and impact of SARS-CoV-2 lineage B.1.1.7 in England. Science 372:eabg305. https://doi.org/10.1126/science.abg30 55 
8. Faria N, Morales I, Candido D et al (2021). Genomic characterization of an emergent SARS-CoV-2 lineage in Manaus: preliminary findings. Virological.org.in. https://bit.ly/3qx9aEU. Accessed 29 Mar 2021

9. Wang P, Casner RG, Nair MS, Wang M, Yu J, Cerutti G, Liu L, Kwong PD, Huang Y, Shapiro L, Ho DD (2021) Increased resistance of SARS-CoV-2 variant P.1 to antibody neutralization. Cell Host Microbe 29:747-751. https://doi.org/10.1016/j.chom.2021. 04.007

10. Lasek-Nesselquist E, Pata J, Schneider E, George K (2021) A tale of three SARS-CoV-2 variants with independently acquired P681H mutations in New York State. MedRxiv. https://doi.org/ 10.1101/2021.03.10.21253285

11. Resende PC, Gräf T, Paixão ACD et al (2021) A potential SARS$\mathrm{CoV}-2$ variant of interest (VOI) harboring mutation E484K in the Spike protein was identified within lineage B.1.1.33 circulating in Brazil. Viruses 13:724. https://doi.org/10.3390/v13050724

12. GISAID (2021) https://www.gisaid.org/. Accessed 29 Mar 2021

13. (2021) CoVsurver: mutation analysis of hCoV-19. https://www. gisaid.org/epiflu-applications/covsurver-mutations-app/. Accessed 29 Mar 2021
14. Starr TN, Greaney AJ, Hilton SK et al (2020) deep mutational scanning of SARS-CoV-2 receptor binding domain reveals constraints on folding and ACE2 binding. Cell 182:1295-1310.e20. https://doi.org/10.1016/j.cell.2020.08.012

15. Liu Z, VanBlargan LA, Bloyet LM et al (2021) Identification of SARS-CoV-2 spike mutations that attenuate monoclonal and serum antibody neutralization. Cell Host Microbe 29:477-488. https://doi.org/10.1016/j.chom.2021.01.014

16. Weekly epidemiological update-25 February 2021. https://www. who.int/publications/m/item/covid-19-weekly-epidemiologicalupdate. Accessed 29 Mar 2021

Publisher's Note Springer Nature remains neutral with regard to jurisdictional claims in published maps and institutional affiliations. 